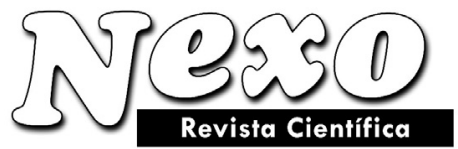

ISSN 1818-6742

Impreso en Nicaragua. www.nexo.uni.edu.ni

Vol. 23, No. 02, pp.47-52/Noviembre 2010

\title{
Control de la Congestión en el IEEE 802.17
}

\author{
E. Gámez*, F. Álvarez
}

Instituto Tecnológico de Acapulco

Departamento de Sistemas y Computación

Av. Instituto Tecnológico S/N, Acapulco, Gro. México

\{egamez, fpaliza\}@it-acapulco.edu.mx

(recibido/received: 24-Agosto-2010; aceptado/accepted: 29-Noviembre-2010)

\section{RESUMEN}

Este trabajo de investigación experimental aborda una solución al problema presentado en el núcleo de las redes Metropolitanas (MAN) por el algoritmo de equidad en modo Agresivo del protocolo 802.17, que sufre de una lenta convergencia para alcanzar el estado estable, lo que genera periodos de oscilaciones dentro de un dominio de congestión.

Palabras Clave: MAN, RPR, Equidad, Congestión.

\begin{abstract}
This experimental research addresses a solution to the problem presented in the core of metropolitan area network (MAN) by an algorithm of equality in an aggressive state of protocol 802.17, which suffers from a slow convergence in order to reach a stable state, generating periods of oscillation within the congested area.
\end{abstract}

Keywords: MAN, RPR, Equality, Congestion.

* Autor para la correspondencia 


\section{INTRODUCCIÓN}

Los estudios sobre la red Ethernet-MAN han originado una gran cantidad de trabajos que comprueban que Ethernet, SONET, WDM o ASON, no son suficientes para resolver el problema de ofrecer calidad del servicio en la red MAN a costos viables. El núcleo de la red MAN debe ofrecer un control eficaz de la congestión, a fin de evitar aspectos negativos que afecten el desempeño de la calidad del servicio de extremo a extremo (retardo, jitter, pérdidas de tramas, disponibilidad), por lo que es esencial establecer un mecanismo que garantice estas métricas de desempeño.

El estándar IEEE 802.17 (RPR) es una solución competitiva para la gestión del tráfico en el núcleo de la red MAN-RPR, puesto que soporta el tráfico de tramas MAC Ethernet, a pesar de esto, RPR sufre de una pérdida de rendimiento a causa de problemas de congestión, pero se comprobó que es posible mejorar el desempeño del control de la congestión perfeccionando el algoritmo de equidad en modo Agresivo de este estándar.

\section{METODOLOGÍA}

\section{Red de anillo de paquetes re-configurable}

En Septiembre del 2004, la IEEE publicó la documentación técnica del estándar 802.17 (2004) conocido también como Resilient Packet Ring (RPR), que define el control de acceso al medio (MAC) para una red MAN óptica de anillo doble y describe la trayectoria y formato de las tramas de datos y control; además ofrece una protección de conmutación automática para la detección de fallas y la correspondiente recuperación de los servicios en el anillo en un tiempo menor a 50 milisegundos 8(Wong, 2003). El estándar 802.17 define que la capa física soporta velocidades de hasta $10 \mathrm{Gbps}$ y que la trama MAC-RPR puede transportar tramas 802.3an, por lo tanto, RPR es una propuesta interesante para extender una red 10 Gigabit Ethernet sobre el núcleo MAN que incluiría características de QoS mediante control de congestión, reutilización espacial, conformación de tráfico y equidad (Khaled, 2006).

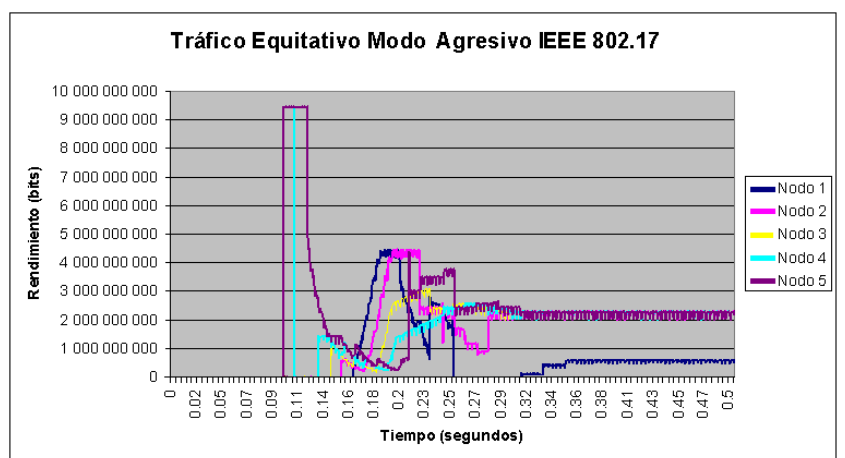

\section{A. Congestión en el núcleo MAN-RPR}

RPR ofrece un conjunto de soluciones aceptables para la gestión del tráfico en la red MAN, pero, en condiciones de congestión severa, se presenta el problema de un bajo rendimiento proporcionado por el algoritmo de equidad RIAS en el modo Agresivo (Davik \& Gjessing, 2005), que se considera necesario para proporcionar control de la congestión, reutilización espacial y distribución equitativa del ancho de banda. El algoritmo de equidad RIAS en modo agresivo produce severas oscilaciones a consecuencia de un mal cálculo de los cambios de los estados de congestión y no congestión en los nodos que forman las cabezas y colas de los dominios de congestión, estos fenómenos son comunes en escenarios sin balanceo de cargas antes que la red pueda alcanzar la estabilidad, ver la figura 1.

Figura 1. Rendimiento y estabilidad del algoritmo de equidad 802.17.

El trabajo de Knightly (2003) describe un modelo de referencia RPR para proporcionar equidad y asegurar la reutilización espacial en la red MAN. En Gjessing (Gjessing, 2001) se reporta el desarrollo de un modelo que se enfoca principalmente a los mecanismos de equidad que operan sobre un anillo MAN. En el trabajo de Kavalbein (Kavalbein, 2003) se describe la mejora del desempeño de un algoritmo para puentes en redes RPR. Robichaud et al (2004) describe un modelo de red de anillo doble que se basa en las especificaciones del 802.17 RIAS, "Agregación de Ingreso al Anillo con Reutilización Espacial”, que utiliza enlaces OC-192. En el trabajo de Gambiroza et al (2004a) se describe un algoritmo de asignación dinámica de ancho de banda llamado DVSR, "Planificador de Tiempo Virtual Distribuido en Anillos". El trabajo presentado por Davik et al (2005), analiza y propone una mejora para resolver el problema generado por las dos principales 
deficiencias en el algoritmo de equidad RPR en modo Agresivo: el cálculo erróneo de la tasa de equidad estimada por el nodo cabeza de un dominio de congestión y la propagación de las notificaciones de equidad, demostrando que el método adoptado por el 802.17 no ofrece estabilidad, produce una lenta convergencia, con grandes periodos de oscilaciones y por lo tanto se obtiene un rendimiento no adecuado.

\section{B. Oscilaciones en el modo Agresivo}

El punto clave que produce las oscilaciones en el modo Agresivo es la tasa de tráfico local a agregar; las tasas de tráfico a enviar son incrementadas periódicamente hasta el punto en que sucede la congestión. Una vez que la congestión ocurre, las tasas de agregación de tráfico de todos los nodos que contribuyen a la congestión son establecidas con el valor de la tasa de agregación mínima; sin embargo, la tasa mínima no es necesariamente la de equidad, en consecuencia, los nodos reducen su tráfico a una tasa por debajo de la de equidad, la congestión se reduce $\mathrm{y}$ los nodos nuevamente inician sus incrementos de tasas. Estas oscilaciones cíclicas continuarán permanentemente bajo ciertas condiciones de tráfico no balanceado y llevarán a una reducción del rendimiento.

Sea $r_{i j}$ la tasa de demanda de flujos (i, j), las condiciones de oscilación del modo Agresivo están dadas por (1 y 2):

Teorema 1: Dada una matriz $\mathrm{R}$ candidata como factible de equidad con demandas $r, y$ un enlace congestionado $\mathrm{j}$, las oscilaciones permanentes ocurrirán en un nodo con el modo Agresivo si existe un flujo (n, i) cruzando el enlace $\mathrm{j}$ siempre que las siguientes dos condiciones sean satisfechas:

$$
(1,3)
$$

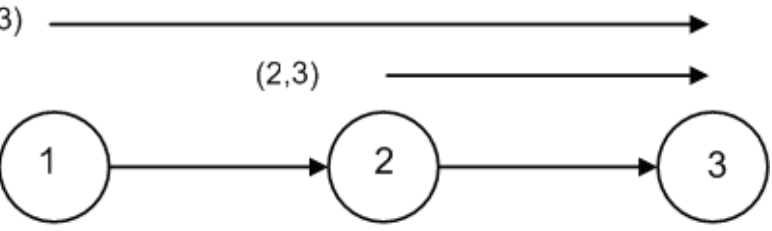

Figura. 2 Escenario de Oscilaciones en nodos RPR.

$$
\begin{gathered}
r_{\text {osc }}=\min _{n<k<j, l>j} \min \left(r_{k l}, R_{k l}\right)<R_{n i} \\
r_{\text {osc }}<R_{n i}
\end{gathered}
$$

Considérese la figura 2, con dos flujos tales que el flujo $(1,3)$ originado ascendentemente tiene una demanda de la capacidad total $=\mathrm{C}$, y el flujo $(2,3)$, originado descendentemente tiene una pequeña tasa de tráfico que se denota por $\varepsilon$. Considérese que el enlace $(1,3)$ sea $\mathrm{j}=2, \mathrm{r}_{\mathrm{osc}}=\varepsilon$ y $\mathrm{R}_{1,3}=\mathrm{C}-\varepsilon$, donde $\mathrm{R}_{13}>\mathrm{r}_{\mathrm{osc}} \mathrm{y}$ $r_{13}>r_{\text {osc }}$. De esta forma las demandas de los flujos son tasas constantes y no balanceadas (figura $3 a$ ).

Dado que la tasa descendente de arribo de tráfico agregado es $\mathrm{C}+\varepsilon$, el enlace descendente se congestionará. Un mensaje de congestión se notificará al nodo ascendente y contendrá la tasa de transmisión del flujo descendente, en este caso $\varepsilon$ (figura $3 b$ ). En consecuencia el nodo ascendente deberá reducir su flujo de la tasa $C$ hasta una tasa $\varepsilon$. En este punto, la tasa en el enlace descendente es 2 veces $\varepsilon$, así que la congestión se elimina (figura 3c). A continuación, el flujo del nodo ascendente incrementará su tasa de nueva cuenta hasta (C- $\varepsilon$ ) una vez recibido el mensaje de no congestión (FULL_RATE). El ciclo se repetirá y la tasa del flujo ascendente oscilará permanentemente entre $(\mathrm{C}-\varepsilon)$ y la tasa del flujo descendente (figura 3d).

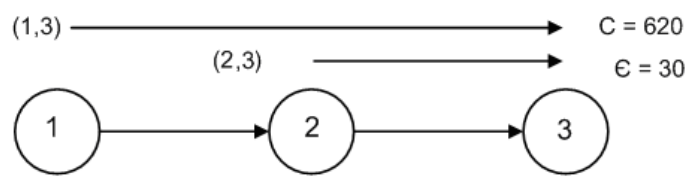

(a)

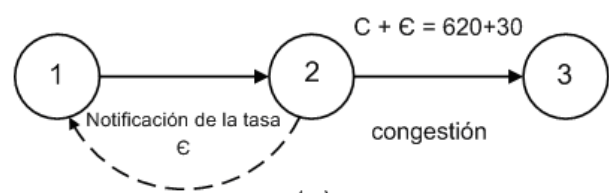

(b)

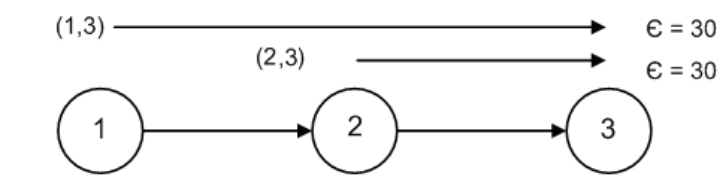

Ajuste de tasa de C hacia $€$

(c)

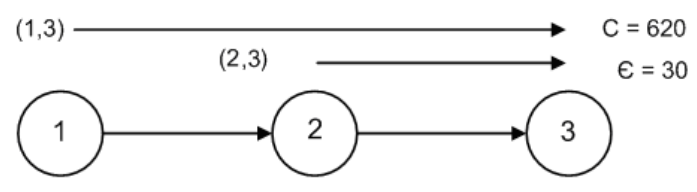

El nodo 1 Incrementa hasta $C$ por lo que la red oscila entre el valor de $C-\epsilon$ y el valor de la tasa descendente $€$

(d)

Fig. 3 Estado de las oscilaciones en una red RPR 
Teorema 2. Considérese una matriz de tasas $\mathrm{R}$ candidatas para equidad, la demanda de flujos $\mathrm{r}$, y un enlace congestionado $\mathrm{j}$, sea el número de flujos activos en el enlace $\mathrm{j}$, y denota el número de flujos que cruzan el enlace $\mathrm{j}$, y que tienen tanto tasas de demanda como tasas $R$ más grandes que $C / n_{a}$. Las oscilaciones permanentes ocurrirán si existe un flujo (n, i) cruzando el enlace $\mathrm{j}$ si las siguientes dos condiciones sean satisfechas:

a) $\min \left(R_{n i}, r_{n}\right)<\frac{C}{n_{a}} \quad \ldots \ldots \ldots \ldots \ldots$ (umbral bajo)
b) $n_{g} \frac{C}{n_{a}}+S_{a}>$ RateLowThreshold (umbral alto)
donde:
\[ S_{S}=\sum_{k \leq j, l>j, \min \left(R_{k l}, r_{k l}\right)<C / n_{a}} \min \left(R_{k l}, r_{k l}\right) \]

El límite inferior del rango de las oscilaciones (3) es $\mathrm{C} / \mathrm{n}_{\mathrm{a}}$ y el límite superior (4) es por debajo del valor del rateLowThreshold y depende de la carga ofrecida de los flujos $\mathrm{n}_{\mathrm{g}}$.

\section{Modificación al modo Agresivo}

En condiciones normales de funcionamiento, un nodo en modo Agresivo notificará una tasa de equidad con un valor igual a su tráfico a agregar localmente (e.g. $30 \mathrm{Mbps})$, de esta forma no existe ningún cálculo de la tasa de equidad y por lo tanto el nodo notifica un valor erróneo, ya que no toma en cuenta el verdadero estado de la capacidad del enlace $C$; de esta forma según el teorema 1, se generarán oscilaciones. Combinando las restricciones del teorema 2 en el modo Agresivo obtenemos un acotamiento del rango de las oscilaciones entre la cantidad de nodos activos (acotamiento bajo $=$ $\mathrm{C} / \mathrm{n}_{\mathrm{a}}=622 / 3=206 \mathrm{Mbps}$ ); el valor del acotamiento alto es el umbral rateLowThreshold $=504$ Mbps.

Primeramente se identifica al nodo ascendente más alejado de la enlace congestionado (la cola) y se calcula el valor de la tasa de equidad en base a un punto de equilibrio definido por el umbral rateLowThreshold, al que se le restará la tasa de tráfico para agregar por el nodo local, y se notificará al nodo ascendente un valor de la tasa de equidad más cercano a la ocupación real del enlace $(504-30=474 \mathrm{Mbps})$, que se encuentre dentro del rango de las restricciones del teorema 2 ( $504>474$ $>206$ ), evitando que el nodo ascendente trate de enviar tráfico a una tasa mayor que la del umbral rateLowThreshold.
Esta técnica de acotación de un intervalo mínimo y máximo, permite disminuir la ganancia de retroalimentación de los mensajes de notificación, ya que se reducirán los ciclos necesarios para llegar a la estabilización, y en cualquier sistema de retroalimentación una disminución de la ganancia implica mayor estabilidad y por lo tanto menor oscilación del sistema.

\section{RESULTADOS Y DISCUSIÓN}

El modelo de red y los algoritmos involucrados en este trabajo se basan en la publicación oficial del estándar IEEE 802.17. La herramienta de simulación utilizada para desarrollar los diversos escenarios, modificaciones hechas al algoritmo de equidad y corridas de simulación fue el OPNET®. En cada experimento de simulación se estimó un nivel de confianza del $95 \%$, con un intervalo de confianza de $\pm 5 \%$ alrededor de la media. Los experimentos llevados a cabo se realizaron teniendo en cuenta las recomendaciones del grupo de trabajo del 802.17 (IEEE, 2007a).

El modelo de red se configuró para un escenario con 6 nodos RPR, interconectados por enlaces ópticos dobles equivalentes a una tasa de transmisión de OC-192. La figura 3.1 representa el modelo utilizado. Esta disposición de nodos y flujos de tráfico se denomina escenario Parking Lot. Cada nodo tiene un valor de ponderación igual a uno, de esta forma todos los nodos de la red tienen una distribución de prioridad justa. Todos los nodos, excepto el CERO, generarán una tasa uniforme de tráfico de $2 \mathrm{Gbps}$, con un tamaño de trama de 1500 bytes. Todo este tráfico se dirigirá hacia el CERO, de tal forma que se desea saturar el enlace UNOCERO. Para el uso de tramas de control y de la tasa de notificación de equidad necesarias para funcionamiento del algoritmo de equidad, se reserva un 5\% del total del ancho de banda, disponiendo del restante $95 \%$ para utilizar en la red (9,5 Gbps).

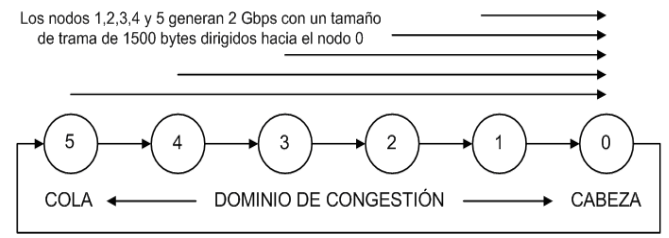

Figura. 4 Nodos de la red MAN RPR. 
En la figura 5 se puede apreciar, como se esperaba, un pico en el rendimiento del ancho de banda en el tiempo de simulación de $\mathrm{t}=0,10$ segundos debido a la sobresaturación de tráfico generada por los nodos de la red, después de este pico se establece un periodo de transición (oscilaciones) antes de que el algoritmo de equidad alcance el estado estable en el $\mathrm{t}=0,20$ segundos, donde se puede apreciar un valor promedio de ajuste de 1,69 Gbps de ancho de banda asignado a cada uno de los cinco nodos participantes en el envío de tráfico sobre la red MAN (rendimiento $=(1,69 \mathrm{Gbps} \bullet 5)$ $=8,46$ Gbps). Lo que demuestra que el algoritmo mejorado logra un rendimiento del $90 \%$ en la utilización del ancho de banda total en situaciones de congestión severa sobre una red de banda ancha.

En la figura 1, se aprecia el rendimiento del algoritmo de equidad 802.17 RIAS. Nótese como el tiempo de convergencia $(t=0,35$ segundos) es mayor al presentado por la variante mejorada, la que logra una reducción del $43 \%$ del tiempo de convergencia. La variación del rango de las oscilaciones es mayor y la distribución del ancho de banda no es equitativa, ya que cuatro flujos se estabilizan a una tasa mayor a 2 Gbps, y un quinto flujo se estabiliza a una tasa promedio de 584 Mbps (vea figura 1).

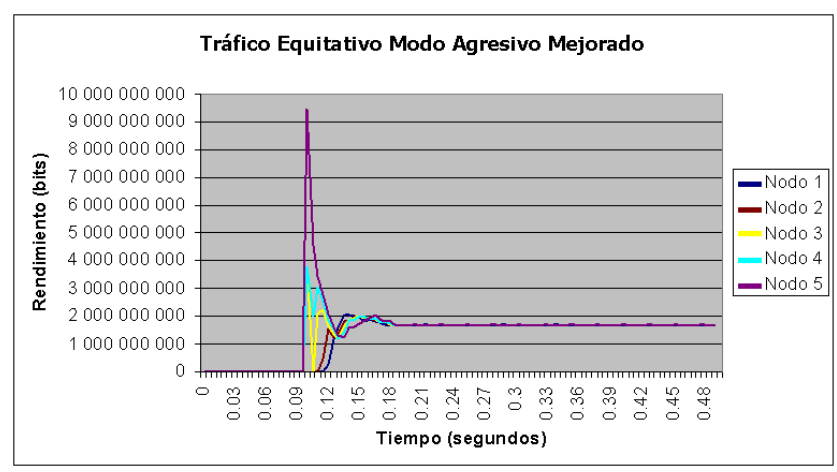

Fig. 5 Rendimiento y estabilidad del algoritmo de equidad perfeccionado.

La tabla 1 muestra el resumen de la matriz de datos obtenida en la simulación del algoritmo de Equidad en modo Agresivo mejorado. Nótese que la oscilación del sistema, en el tiempo de simulación con la mayor estabilidad, se encuentra dentro del rango de 0,20 a 0,49 segundos. La diferencia máxima de la variación del rango de las oscilaciones de los flujos equitativos se puede calcular con el valor promedio del rango de oscilación mínimo y máximo (1 691030400 bits); para el caso de los flujos del algoritmo mejorado, la diferencia máxima del promedio es de $0,2163 \%$; para el caso de los flujos del 802.17 RIAS no existe equidad en los cinco flujos por lo que no se realiza el cálculo.

Tabla 1. Oscilaciones del algoritmo de equidad mejorado

RANGO DE OSCILACIONES EN EL ALGORITMO DE EQUIDAD IEEE 802.17 RIAS

\begin{tabular}{cccc}
\hline \hline Nodo & $\begin{array}{c}\text { Tasa Mínima } \\
\text { (bits) }\end{array}$ & $\begin{array}{c}\text { Tasa Máxima } \\
\text { (bits) }\end{array}$ & $\begin{array}{c}\text { Variación del Rango de Oscilación } \\
\text { (bits) }\end{array}$ \\
\hline 1 & 560832000 & 609600000 & 48768000 \\
\hline 2 & 1999488000 & 2292096000 & 292608000 \\
\hline 3 & 1999488000 & 2267712000 & 268224000 \\
\hline 4 & 1999488000 & 2292096000 & 292608000 \\
\hline 5 & 1999488000 & 2292096000 & 292608000 \\
\hline
\end{tabular}

El nodo CUATRO presenta la variación del rango de oscilación más alta del algoritmo mejorado, con un valor máximo de 7315200 bits, este rango es 97.5\% menor que en el ofrecido por el modo Agresivo RIAS; las oscilaciones en el modo mejorado son menos drásticas que las obtenidas por el 802.17 RIAS donde varios nodos presentan una máxima variación del rango de oscilación de 292608000 bits; ver la tabla 2 .

Tabla 2. Rango de Oscilaciones en el algoritmo Equidad IEEE 802.17 RIAS.

\begin{tabular}{cccccc}
\multicolumn{5}{c}{ RANGO DE OSCILACIONES EN EL ALGORITMO DE EQUIDAD MEJORADO } \\
\hline \hline Nodo & $\begin{array}{c}\text { Tasa Mínima } \\
\text { (bits) }\end{array}$ & $\begin{array}{c}\text { Diferencia } \\
\text { Mínima } \\
(\%)\end{array}$ & $\begin{array}{c}\text { Tasa Máxima } \\
\text { (bits) }\end{array}$ & $\begin{array}{c}\text { Diferencia } \\
\text { Máxima } \\
(\%)\end{array}$ & $\begin{array}{c}\text { Variación del Rango } \\
\text { de Oscilación } \\
\text { (bits) }\end{array}$ \\
\hline 1 & 1689811200 & 0,0721 & 1694688000 & 0,2163 & 4876800 \\
\hline 2 & 1689811200 & 0,0721 & 1694688000 & 0,2163 & 4876800 \\
\hline 3 & 1692249600 & 0,0721 & 1694688030 & 0,2163 & 2438430 \\
\hline 4 & 1687372800 & 0,2163 & 1694688000 & 0,2163 & 7315200 \\
\hline 5 & 1687372800 & 0,2163 & 1692249600 & 0,0721 & 4876800 \\
\hline
\end{tabular}

\section{CONCLUSIONES}

Se demostró que la modificación al algoritmo de equidad en modo Agresivo del estándar IEEE 802.17, es un mecanismo de gestión de capa MAC que ofrece una nueva variante para enfrentar y minimizar los problemas de la congestión del tráfico en los nodos que integran el núcleo de la red MAN-RPR. La mejora del algoritmo de equidad, permite obtener una convergencia más rápida ( $\mathrm{t}=0,20$ segundos), $\mathrm{y}$ periodos de inestabilidad menores a los referenciados por el estándar IEEE 802.17 RIAS $(\mathrm{t}=0,35$ 
segundos) y en trabajos de investigación similares. La mejora del algoritmo de equidad minimiza los problemas de congestión al reducir la variación del rango de las oscilaciones en un $97,5 \%$, y reducir el tiempo de convergencia en un $43 \%$. El algoritmo mejorado también ofrece un rendimiento de la utilización efectiva del ancho de banda del 90\%, logrando asignar a todos los flujos activos tasas equitativas con una diferencia máxima del 0,2163\% entre un flujo y otro.

\section{REFERENCIAS}

Davik, F. \& Gjessing, S. (2005) "Improvement of Resilient Packet Ring Fairness". The 48th annual IEEE Global Telecommunications Conference, GLOBECOM 2005. pp 1-8.

Gambiroza, V., Yuan, P., Balzano, L., Liu, Y., Sheafor, S. \& Knightly, E. (2004a) "Design, analysis, and implementation of DVSR: a fair highperformance protocol for packet rings". IEEE/ACM Transactions on Networking, 2, pp 85-102.

Gjessing, S. (2001) "Proyecto DYNARC", Universidad de Oslo, Noruega. http://www.simula.no/photo/sim1mars.pdf.

IEEE (2004) Std 802.17, Resilient Packet Ring (RPR) Access Method and Physical Layer Specifications

IEEE (2007a) IEEE 802.17 Working group, "Guidance for IEEE 802.17 RPR Performance Simulations", http://www.ieee802.org/17/performance_committee.h tm.

Kavalbein, A. (2003) "Bridging in RPR Networks: Evaluation of an Enhanced Bridging Algorithm". Department of Informatics. Oslo, Noruega, University of Oslo, pp 120.

Khaled, M. F. (2006) "Overview of the Evolving IEEE 802.17 Resilient Packet Rings Standard". Department of Electronics and Communications Engineering. Giza, Egypt, Cairo University.

Knightly, E. (2003) "Proyecto RIAS", Universidad RICE, Texas, USA. http://www.ece.rice.edu/networks/RIAS.
Robichuad, Y., Huang, C., Yang, J. \& Peng, H. (2004) "Access Delay Performance of resilient Packet Ring under Bursty Periodic Class B Traffic Load". IEEE International Conference on Communications. pp $1217-1221$.

Wong, Y. F. (2003) "Operation, Management and Performance Isssues of LAN Technologies Applied to WAN Architecture". Eighth IEEE International Symposium on Computers and Communication (ISCC 2003), Junio 2003. National University of Singapore.

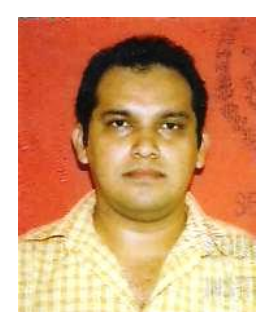

Profesor investigador del Instituto Tecnológico de Acapulco (1998). Ingeniero en Sistemas Computacionales (1997) y Doctor en Ciencias de la Computación por la universidad Central Marta Abreu de las Villas, República de Cuba (2008). Director de la Carrera de Ingeniería en Computación de la Universidad Americana de Acapulco (2008). Miembro de la IEEE (2005) y de RIEI. Ha publicado artículos de divulgación cientifica en revistas Internacionales y tambien ha participado como ponente en eventos Internacionales (2003 ala fecha). Técnico de la revista científica NEXO de la Universidad Nacional de Nicaragua (2010). La actual línea de investigación es en modelos de simulación en redes de datos de alta velocidad y seguridad informática en servicios web Revisor. 